\title{
Die Agenda 2030 und die Sustainable Development Goals
}

Im Jahr 2015 verabschiedeten die Mitgliedsstaaten der Vereinten Nationen die Agenda 2030 für nachhaltige Entwicklung. Die Agenda als globales Aktionsprogramm sieht bis zum Jahr 2030 weltweit gemeinsame Anstrengungen vor, um die Sustainable Development Goals (SDGs) zu erreichen. Die Realisierung dieser ambitionierten globalen Nachhaltigkeitsziele erscheint angesichts neuer Herausforderungen wie z. B. dem Umgang mit den globalen Auswirkungen der COVID-19 Pandemie schwierig.

Gleichzeitig werden die Effekte des globalen Umweltwandels immer deutlicher und die Notwendigkeit eines schonenderen Umgangs mit Ressourcen gewinnt in der Gesellschaft an Bedeutung. Exemplarisch hierfür steht der Klimawandel und die Protestbewegung Fridays for Future. Weitere große Herausforderungen wie z. B. dem Verlust der Biodiversität entgegenzusteuern oder den Eintrag von Mikroplastik in den Ozeanen zu reduzieren, verdeutlichen die Dringlichkeit zum Handeln.

Was genau steht in der Agenda 2030 und den SDGs, wie kann hierdurch eine nachhaltige Entwicklung weltweit vorangetrieben werden und welche Rolle kommt den Städten und Gemeinden bei der Umsetzung zu?

\section{Definitionen von Nachhaltigkeit}

Im ursprünglichen Sinn wird unter Nachhaltigkeit ein ressourcen-ökonomisches Prinzip verstanden, durch das eine Ressource dauerhaft ertragsbringend genutzt werden kann (Pufé 2017). Ausgehend von der Forstwirtschaft hat sich der Begriff zunehmend auch für komplexere Fragestellungen als Leitvorstellung durchgesetzt, um angesichts endlicher natürlicher Ressourcen die Bedürfnisse jetziger sowie künftiger Generationen in gleichem Maße befriedigen zu können. Hierzu ist das Zusammenspiel der Dimensionen Ökologie, Ökonomie und Soziales notwendig. Das Nachhaltigkeitsdreieck, in dem diese Dimensionen gleichberechtigt miteinander verbunden sind, verdeutlicht die Notwendigkeit einer integrierten Sichtweise.

F. Koch und K. Krellenberg, Nachhaltige Stadtentwicklung, essentials, https://doi.org/10.1007/978-3-658-33927-2_2 
Auch die Agenda 2030 und die SDGs bauen auf diesem Ansatz auf. Eine Übersicht zu generellen Definitionen von Nachhaltigkeit, zum Entstehungskontext und zur Umsetzung von Nachhaltigkeit in Politik, Wirtschaft und Gesellschaft findet sich in Grunwald und Kopfmüller (2012) oder Pufé (2017).

\subsection{Entstehungsgeschichte}

Zunächst empfiehlt sich ein Blick auf die den SDGs vorangegangen sogenannten Millenium Development Goals (MDGs), auf die sich die Mitgliedsstaaten der Vereinten Nationen im Jahr 2000 einigten. Die MDGs gliederten sich in acht Unterziele und umfassten Themen wie die Bekämpfung von Hunger und Armut, die Senkung der Kindersterblichkeit und die Gleichstellung der Geschlechter (Hulme 2009).

Mit den MDGs sollten Anstrengungen unternommen werden, um bis zum Jahr 2015 die (absolute) Armut zu beenden. Im Fokus standen die Entwicklungsländer, die sogenannten Länder des Globalen Südens. Somit stellten die MDGs einen Orientierungsrahmen der globalen Entwicklungszusammenarbeit dar.

Für eine Überprüfung der Erfüllung der Ziele wurden verschiedene Zielvorgaben gemacht. So sollte beispielsweise zwischen 1990 und 2015 weltweit die Sterblichkeitsrate von Kindern unter fünf Jahren unter anderem durch Impfungen um zwei Drittel gesenkt werden. Als Indikator wurde dafür zum Beispiel der Anteil der Einjährigen, die gegen Masern geimpft wurden, herangezogen.

Die nachhaltige Entwicklung von Städten spielte bei den MDGs keine explizite Rolle. Durch Ziele wie beispielsweise die Beseitigung von Slums bestanden jedoch indirekte Bezüge zur Stadtentwicklung. Auch die Indikatoren wurden in der Regel nicht auf lokaler Ebene, sondern auf nationaler bzw. supranationaler Ebene gemessen.

Obwohl von allen UN-Mitgliedsstaaten unterzeichnet, entfalteten die MDGs keine unmittelbaren völkerrechtlichen Konsequenzen für staatliches Handeln. Durch zeitliche Vorgaben und Indikatoren zur Messung der Umsetzung bestand jedoch Handlungsdruck. Für einige der Ziele konnten bis zum Jahr 2015 auch durchaus Fortschritte verzeichnet werden: So sank die Zahl der Menschen, die in extremer Armut leben, von 1,9 Mrd. im Jahr 1990 auf 836 Mio. im Jahr 2015. Die Kindersterblichkeit nahm im selben Zeitraum von 90 auf 43 Tote pro 1000 Geburten ab (United Nations 2015a). Es gab jedoch auch Kritik an den MDGs: fehlende Daten zur Messung der Indikatoren, begrenzte Einbeziehung der Länder 
des Globalen Südens bei der Entwicklung der MDGs und keine Anpassung an spezifische nationale Begebenheiten (Attaran 2005; Fehling et al. 2013).

Angesichts der Erfahrungen mit den MDGs starteten die Vereinten Nationen im Jahr 2012 den sogenannten Post-2015-Prozess, in dem neue globale Entwicklungsziele diskutiert wurden. Während des Prozesses fanden Konsultationen mit der Zivilgesellschaft, dem Privatsektor und den Regierungen einer großen Zahl der UN Mitgliedstaaten statt. Auch die Wissenschaft wurde einbezogen (International Council for Science ICSU und International Social Science Council ISSC 2015). Partizipation spielte somit im Post-2015 Prozess eine wesentlich größere Rolle als bei der Erstellung der MDGs (Feeny 2020). Trotz eines konfliktbeladenen Prozesses, in dem unterschiedliche Stakeholder und Lobbygruppen um Einfluss kämpften, sowie harter Diskussionen um die Ziele (Kamau et al. 2018; Parnell 2016), gelang es der UN Vollversammlung im September 2015 sich mit der Agenda 2030 und den darin enthaltenen 17 SDGs auf ein komplexes umfassendes Zielsystem zu einigen: Innerhalb von 15 Jahren (2015-2030) sollen mit den SDGs umfassende Maßnahmen hin zu einer globalen nachhaltigen Entwicklung realisiert werden. Die Ambitionen der MDGs werden damit deutlich übertroffen. Im Kern geht es darum, die Welt zu transformieren, wie es der Untertitel der Agenda 2030 beschreibt: „Transforming our world“. Im Vergleich zu den MDGs verfügen die SDGs über:

- Umfangreichere Ziele: Während der Fokus der MDGs auf klassischen entwicklungspolitischen Zielen lag, umfassen die SDGs neben der Armutsbekämpfung (SDG 1) weitere nachhaltigkeitsbezogene Ziele wie z. B. Maßnahmen zum Klimaschutz (SDG 13), die Gleichstellung der Geschlechter (SDG 5) oder bezahlbare und saubere Energie (SDG7).

- Ein globales Transformationsverständnis: Die MDGs legten Ziele für die Länder des Globalen Südens fest, die durch eine gemeinsame Kraftanstrengung von reichen und armen Ländern erreicht werden sollten. Den SDGs liegt hingegen ein globales Verständnis notwendiger Transformationen zugrunde. Für die Erreichung der Ziele sollen sich auch die reichen Industrieländer, die Länder des Globalen Nordens, nachhaltiger entwickeln. Das bedeutet, dass Transformationen in allen Ländern notwendig sind und alle Länder „Entwicklungsländer“ hin zur Nachhaltigkeit sind.

- Einen städtischen Bezug: Die Zielebene der MDGs war die Länderebene, d. h. es wurde gemessen, inwieweit bestimmte Ziele wie z. B. die Kindersterblichkeit zu reduzieren in einem spezifischen Land erreicht wurde. Die SDGs hingegen sind nicht nur im Landesdurchschnitt zu erreichen, sondern auch auf regionaler und lokaler Ebene. Zudem gibt es mit SDG 11 (Nachhaltige Städte 
und Gemeinden) ein SDG, das sich explizit auf die nachhaltige Entwicklung von Städten und Gemeinden bezieht, was die Bedeutung der Städte für die Erreichung globaler Nachhaltigkeitsziele untermauert.

- Das Thema der nachhaltigen Stadtentwicklung ist somit prominent in der Agenda 2030 vertreten und als Teil globaler politischer Nachhaltigkeitsprozesse anerkannt. Die Äußerung des früheren UN Generalsekretärs Ban Ki Moon „Our struggle for Global Sustainability will be won or lost in Cities“ (Unser Kampf für globale Nachhaltigkeit wird in den Städten gewonnen oder verloren werden) (United Nations 2012) verdeutlicht diese Verschiebung hin zu einem städtischen Fokus globaler Politik.

\subsection{Ziele, Unterziele und Indikatoren}

In diesem Kapitel werden wir näher auf die Inhalte und den strukturellen Aufbau der Agenda 2030 und die SDGs eingehen, um in Kap. 3 die Bedeutung für die nachhaltige Stadtentwicklung genauer zu beleuchten. Die 17 Ziele (Abb. 2.1) bilden unterschiedliche Themenfelder $a b$ und bauen auf existierenden

\section{THEGLOBAL GOALS \\ DieglobalenZiele fürnachhaltige Entwicklung}
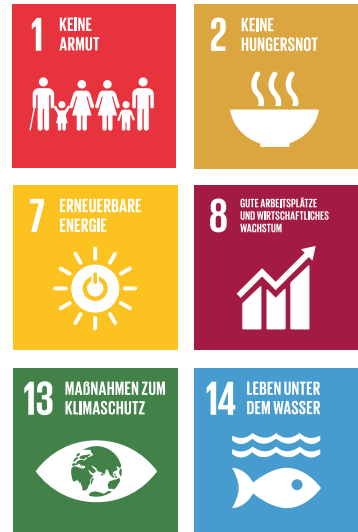

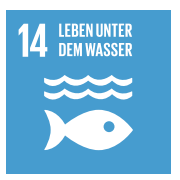

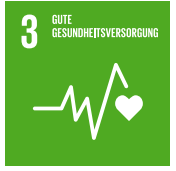
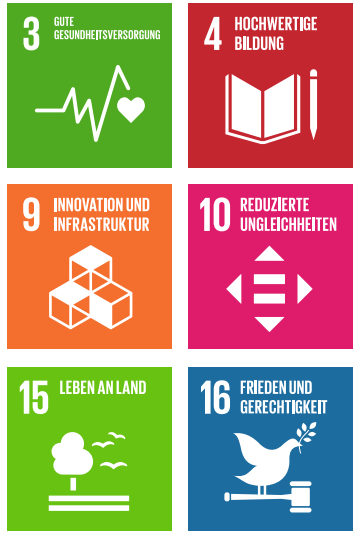
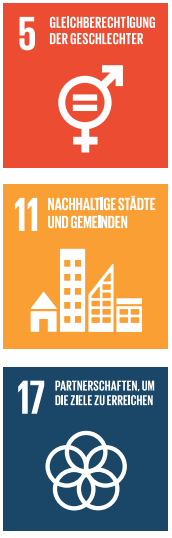
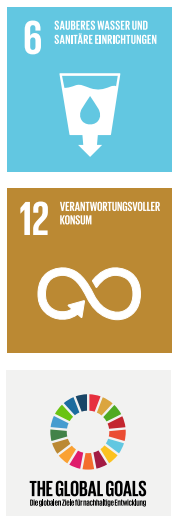

Abb. 2.1 Die 17 SDGs (https://unric.org/de/17ziele/) 
Nachhaltigkeitsdefinitionen wie dem Nachhaltigkeitsdreieck mit den Dimensionen Ökonomie, Ökologie und Soziales auf. Sie zielen darauf, die Lebensgrundlage jetziger und künftiger Generationen sicherzustellen.

Die einzelnen 17 SDGs sind nicht priorisiert, sondern stehen nebeneinander und sollen sich gegenseitig ergänzen. Diesen 17 eher generell gehaltenen Zielen wurden jeweils 8-12 Unterziele zur Konkretisierung zugeordnet, die über Indikatoren gemessen werden können. Hierdurch soll erreicht werden, dass die Umsetzung der SDGs evaluiert werden kann, indem transparent und nachvollziehbar dargestellt wird, welche Fortschritte hin zur Erreichung der Ziele gemacht wurden. Den 17 Zielen wurden insgesamt 169 Unterziele zugeordnet, die durch 231 Indikatoren gemessen werden sollen (United Nations 2015b).

- SDG-Ziele, Unterziele und Indikatoren Eine vollständige Übersicht zu den Zielen, Unterzielen und den entsprechenden Indikatoren der Vereinten Nationen finden Sie auf den Internetseiten der Statistikabteilung der Vereinten Nationen (UNSD 2021): https://unstats.un.org/sdgs/indica tors/indicators-list/.

Ziel von SDG 11 ist es, Städte und Siedlungen inklusiv, sicher, widerstandsfähig und nachhaltig zu gestalten. Damit wurde ein integratives Verständnis von Stadtentwicklung mit verschiedenen, miteinander verwobenen Zielen in einem Dokument der globalen Politik verankert (Parnell 2016). SDG 11 kann daher in Verbindung mit den anderen SDGs, die einen städtischen Bezug aufweisen, als globaler Rahmen für nachhaltige Stadtentwicklung dienen, auf den sich alle UN Mitgliedsstaaten im Konsens geeinigt haben.

Auch SDG 11 sind verschiedene Unterziele zugeordnet. Hierzu zählen die urbane Mobilität, der öffentliche Raum, Flächennutzung, die Widerstandsfähigkeit von Städten gegenüber Katastrophen, sowie dezidiert eine partizipative, integrierte und nachhaltige Stadtplanung. Es finden sich folglich mehrere Dimensionen nachhaltiger Stadtentwicklung in diesem Ziel wieder (vgl. auch Kap. 3). Somit wird nicht nur eine ökologischere Form der Stadtentwicklung angestrebt, sondern soziale, kulturelle und wirtschaftliche Ziele werden gleichermaßen verfolgt.

\section{Unterziele von SDG 11}

Eine Übersicht zu den Daten für Deutschland findet sich auf der Seite des Statistischen Bundesamts (Statistisches Bundesamt 2021a).

- Unterziel 11.1: Bis 2030 den Zugang zu angemessenem, sicherem und bezahlbarem Wohnraum und zur Grundversorgung für alle sicherstellen und Slums sanieren 
- Indikator 11.1.1: Anteil der städtischen Bevölkerung, der in Slums, informellen Siedlungen oder unzureichendem Wohnraum lebt

- Unterziel 11.2: Bis 2030 den Zugang zu sicheren, bezahlbaren, zugänglichen und nachhaltigen Verkehrssystemen für alle ermöglichen und die Sicherheit im Straßenverkehr verbessern, insbesondere durch den Ausbau des öffentlichen Verkehrs, mit besonderem Augenmerk auf den Bedürfnissen von Menschen in prekären Situationen, Frauen, Kindern, Menschen mit Behinderungen und älteren Menschen

- Indikator 11.2.1: Anteil der Bevölkerung mit angemessenem Zugang zu öffentlichen Verkehrsmitteln, nach Geschlecht, Alter und Menschen mit Behinderungen

- Unterziel 11.3: Bis 2030 die Verstädterung inklusiver und nachhaltiger gestalten und die Kapazitäten für eine partizipatorische, integrierte und nachhaltige Siedlungsplanung und -steuerung in allen Ländern verstärken

- Indikator 11.3.1: Verhältnis der Flächennutzungs- zur Bevölkerungswachstumsrate

- Indikator 11.3.2: Anteil der Städte mit einer regelmäßig und demokratisch arbeitenden direkten Beteiligungsstruktur der Zivilgesellschaft an der Stadtplanung und -verwaltung

- Unterziel 11.4: Die Anstrengungen zum Schutz und zur Wahrung des Weltkultur- und -naturerbes verstärken

- Indikator: 11.4.1 Gesamtausgaben pro Kopf für die Erhaltung und den Schutz des gesamten Kultur- und Naturerbes, nach Finanzierungsquelle (öffentlich, privat), Art des Erbes (Kulturerbe, Naturerbe) und Verwaltungsebene (national, regional, lokal/kommunal)

- Unterziel 11.5: Bis 2030 die Zahl der durch Katastrophen, einschließlich Wasserkatastrophen, bedingten Todesfälle und der davon betroffenen Menschen deutlich reduzieren und die dadurch verursachten unmittelbaren wirtschaftlichen Verluste im Verhältnis zum globalen Bruttoinlandsprodukt wesentlich verringern, mit Schwerpunkt auf dem Schutz der Armen und von Menschen in prekären Situationen

- Indikator 11.5.1: Anzahl der Katastrophen zugeschriebenen Todesopfer, Vermissten und direkt Betroffenen je 100.000 Einwohner

- Indikator 11.5.2: Katastrophen zugeschriebene direkte wirtschaftliche Schäden im Verhältnis zum globalen Bruttoinlandsprodukt (BIP), Schäden an kritischen Infrastrukturen und Zahl der Unterbrechungen der Grundversorgung

- Unterziel 11.6: Bis 2030 die von den Städten ausgehende Umweltbelastung pro Kopf senken, unter anderem mit besonderer Aufmerksamkeit auf der Luftqualität und der kommunalen und sonstigen Abfallbehandlung

- Indikator 11.6.1: Anteil der in kontrollierten Einrichtungen gesammelten und behandelten festen Siedlungsabfälle an den gesamten Siedlungsabfällen, nach Städten

- Indikator 11.6.2: Bevölkerungsgewichtete Jahresmittelwerte der Feinstaubkonzentration (z. B. $\mathrm{PM}_{2,5}$ und $\mathrm{PM}_{10}$ ) in Städten

- Unterziel 11.7: Bis 2030 den allgemeinen Zugang zu sicheren, inklusiven und zugänglichen Grünflächen und öffentlichen Räumen gewährleisten, insbesondere für Frauen und Kinder, ältere Menschen und Menschen mit Behinderungen

- Indikator 11.7.1: Durchschnittlicher Anteil der bebauten Fläche in Städten, der für alle Personen nach Geschlecht, Alter und Menschen mit Behinderungen, als Freifläche öffentlich zugänglich ist

- Indikator 11.7.2: Anteil der Personen, die in den vorangegangenen 12 Monaten Opfer körperlicher oder sexueller Belästigung wurden, nach Geschlecht, Alter, Behinderungsstatus und Tatort 
- Unterziel 11.a: Durch eine verstärkte nationale und regionale Entwicklungsplanung positive wirtschaftliche, soziale und ökologische Verbindungen zwischen städtischen, stadtnahen und ländlichen Gebieten unterstützen

- Indikator: 11.a.1: Anzahl der Staaten, die über eine nationale Städtepolitik oder regionale Entwicklungsplanung verfügen, welche a) auf die Bevölkerungsdynamik reagiert, b) eine ausgewogene Raumentwicklung gewährleistet und c) den lokalen Haushaltsspielraum vergrößert

- Unterziel 11.b: Bis 2020 die Zahl der Städte und Siedlungen, die integrierte Politiken und Pläne zur Förderung der Inklusion, der Ressourceneffizienz, der Abschwächung des Klimawandels, der Klimaanpassung und der Widerstandsfähigkeit gegenüber Katastrophen beschließen und umsetzen, wesentlich erhöhen und gemäß dem Sendai-Rahmen für Katastrophenvorsorge 2015-2030 ein ganzheitliches Katastrophenrisikomanagement auf allen Ebenen entwickeln und umsetzen

- Indikator 11.b.1: Anzahl der Staaten, die nationale Strategien zur Katastrophenvorsorge im Einklang mit dem Sendai-Rahmenwerk für Katastrophenvorsorge 2015-2030 beschließen und umsetzen

- Indikator 11.b.2: Anteil der Gemeinden, die lokale Strategien zur Katastrophenvorsorge im Einklang mit nationalen Strategien zur Katastrophenvorsorge beschließen und umsetzen

$\mathrm{Zu}$ beachten ist, dass das Konzept der Agenda 2030 mit den SDGs insbesondere die Verknüpfung von Sektoren hervorhebt und systemische Zusammenhänge zwischen den verschiedenen SDGs betont (Beisheim 2018). Für die Umsetzung von SDG 4 ,Hochwertige Bildung“ oder SDG 6 ,Sauberes Wasser und sanitäre Einrichtungen" sind beispielsweise städtische Maßnahmen von besonderer Bedeutung, da sich in den Städten die Bevölkerung konzentriert (Koch et al. 2019). Auch zwischen SDG 7 „Erneuerbare Energie“ und SDG 11 gibt es enge Verbindungen (International Council for Science ICSU 2017). So ist angesichts steigender Urbanisierungsquoten und zunehmender städtischer Energieverbräuche die Frage zentral, wie in den Städten eine nachhaltigere Form der Energieversorgung erreicht werden kann. UN HABITAT, das Programm der Vereinten Nationen für menschliche Siedlungen, geht davon aus, dass ein Drittel aller Indikatoren für die SDGs sich auch auf urbaner Ebene messen lassen (UN Habitat 2018).

Ebenfalls zu beachten ist, dass die SDGs zwar einen universellen Anspruch haben, die einzelnen Ziele jedoch in Ländern des Globalen Nordens und Südens unterschiedliche Bedeutung haben. So ist in Bezug auf SDG 1 „Keine Armut" in Ländern des Globalen Nordens das Thema der relativen Armut anzusprechen, während im Globalen Süden auch die absolute Armut verringert werden soll (Koch et al. 2019). Auch von Stadt zu Stadt kann sich der Bezug und die Ausrichtung der jeweiligen SDGs ändern, da Städte spezifische Charakteristika und Herausforderungen bezüglich einer nachhaltigen Stadtentwicklung aufweisen. Insofern ist es notwendig, die universell gültigen Ziele jeweils kontextspezifisch 
zu prüfen und gegebenenfalls anzupassen (Krellenberg et al. 2019; Koch et al. 2019).

\subsection{Umsetzungsinstrumente der Agenda 2030}

Die Agenda 2030 hat keine unmittelbare Rechtswirksamkeit. Somit sind auch die SDGs rechtlich nicht bindend. Insofern besteht die offensichtliche Gefahr, dass die SDGs als unverbindliche Absichtserklärung (,Schönwetterstrategie“) nicht die notwendige Wirkung erzielen, die für das Erreichen der ambitionierten Nachhaltigkeitsziele notwendig wäre. Angesichts dieser Situation haben die Vereinten Nationen einige Instrumente und Verfahren etabliert, die die Umsetzung der Ziele unterstützen sollen. Der dafür gewählte Ansatz wird von Biermann et al. (2017) als Governance through goals (Steuerung durch Ziele) bezeichnet, der vornehmlich auf Freiwilligkeit setzt. Zentral ist in diesem Zusammenhang das neu gegründete High-Level Political Forum (HLPF). Die einzelnen Länder und andere Akteure können das HLPF auf freiwilliger Basis nutzen, um ihre Fortschritte bei der Umsetzung der SDGs in Voluntary National Reviews (VNR) vorzustellen.

\section{High-Level Political Forum}

Das High-Level Political Forum on Sustainable Development (HLPF) ist das entscheidende Gremium der Vereinten Nationen zur Abstimmung der globalen Nachhaltigkeitspolitik und somit auch zur Überprüfung der Agenda 2030. Das HLPF hat verschiedene Aufgaben (vgl. Beisheim 2018): Auf dem HLPF werden die sogenannten Voluntary National Reviews (VNR) vorgestellt, die freiwilligen nationalen Berichte, in denen die Mitgliedsstaaten die Umsetzung der SDGs in ihren jeweiligen Ländern vorstellen. Auch werden auf dem jährlich stattfindenden HLPF thematische Reviews durchgeführt, in denen die globale Umsetzung einzelner SDGs überprüft sowie der SDG Progress Report des UN-Generalsekretärs (United Nations 2020) vorgestellt wird. Im Jahr 2018 wurde auf dem HLPF beispielsweise ein Bericht zur Umsetzung von SDG 11 veröffentlicht (UN Habitat 2018).

Das HLPF soll ebenfalls eine Plattform für nicht-staatliche Akteure, wie z. B. auch Vertreter von Städten und Städtenetzwerken sein. Alle vier Jahre wird darüber hinaus der Global Sustainable Development Report von einem hierzu ernannten Team internationaler Forscher*innen auf dem HLPF vorgestellt (Independent Group of Scientists appointed by the Secretary-General 2019). Allerdings zeigt sich, dass bislang nicht alle Erwartungen an das HLPF erfüllt werden konnten, was unter anderem an den geringen zur Verfügung stehenden Ressourcen liegt (Beisheim \& Stiftung Wissenschaft und Politik 2019). In Deutschland liegt die Federführung für das HLPF gemeinsam beim Bundesministerium für Umwelt, Naturschutz und nukleare Sicherheit (BMU) und beim Bundesministerium für wirtschaftliche Zusammenarbeit und Entwicklung (BMZ). 
Die Umsetzung der SDGs wird also nicht als top-down Prozess, sondern als bottom-up Verfahren verstanden (Biermann et al. 2017). Da keine klaren Vorgaben und Sanktionen vorliegen, erlauben die SDGs den einzelnen Ländern viel Spielraum in der Ausgestaltung der eigenen nationalen Schwerpunktsetzung bei der Umsetzung der SDGs. Die SDGs erfordern nationale und auch lokale Interpretationen und Umsetzungen, wie in den folgenden Kapiteln erläutert wird. Aber auch in Bezug auf Maßnahmen zur Umsetzung der Ziele auf nationaler und lokaler Ebene enthält die Agenda 2030 nur wenige Aussagen. Somit bestehen in Bezug auf die nationalen Berichtswesen erhebliche Unterschiede zwischen den Mitgliedstaaten (Beisheim 2020).

Unklar ist, wer die Verantwortung (accountability) für die Umsetzung trägt und was passiert, wenn in einzelnen Ländern die entsprechend der SDGs selbst gesteckten Ziele nicht erreicht werden (Bowen et al. 2017). Vor dem Hintergrund, dass die 17 Ziele umfassend und komplex sind, scheint es nicht möglich, dass staatliche Akteure allein für die Umsetzung der Agenda 2030 zuständig sind. Vielmehr müssen verschiedene Akteure aus Wirtschaft und Zivilgesellschaft bei der Umsetzung der Ziele beteiligt werden. Wie genau dieser Einbezug nichtstaatlicher Akteure erfolgt, ist im Dokument der Agenda 2030 allerdings nur sehr vage formuliert. So finden sich beispielsweise keine Hinweise darauf, wie Daten zivilgesellschaftlicher Akteure oder von Unternehmen eingebracht und zu einem SDG-Monitoring beitragen können.

Zwar betont die Agenda 2030 die wichtige Rolle der lokalen Ebene bei der Umsetzung der SDGs, genaue Verfahren bzw. verpflichtende Zielkataloge für Städte existieren hingegen nicht. Für die Städte ergeben sich daher aus der Umsetzungsarchitektur der Agenda 2030 große Freiheiten, aber auch Herausforderungen (siehe Kap. 3).

\subsection{Widersprüche und Kritik}

Die Agenda 2030 hat einige der Probleme, die bei den MDGs auftraten, gelöst. Mit den SDGs wird ein universeller Anspruch verfolgt und die Spannbreite der Nachhaltigkeitsaspekte ist größer. Allerdings existiert auch Kritik an den SDGs. Zielkonflikte, nicht ausreichend große Ambitionen bei der Umsetzung sowie Zweifel an der Wirksamkeit der Ziele werden in diesem Zusammenhang genannt und im Folgenden näher erläutert.

Da die Agenda 2030 keine Priorisierung der 17 SDGs vorsieht, sind alle SDGs gleichbedeutend und stehen auf einer Ebene. Im Idealfall ergeben sich Synergieeffekte, wie in Abschn. 2.2 bereits am Beispiel von SDG 11 aufgezeigt wurde: 
Maßnahmen, die ein SDG betreffen, können gleichzeitig zur Erfüllung eines anderen SDGs beitragen. Allerdings birgt dies auch die Gefahr, dass Zielkonflikte - sogenannte Trade-offs - zwischen den Zielen auftreten. Durch eine Intensivierung des Fischfangs mag es zwar gelingen, Hunger zu reduzieren (SDG 2), gleichzeitig können jedoch auch negative Auswirkungen auf das Leben unter Wasser (SDG 14) entstehen. Das in SDG 8 erwähnte Ziel des Wirtschaftswachstums kann aufgrund der damit in der Regel verbundenen höheren $\mathrm{CO}_{2}$-Emissionen den Klimawandel verstärken und somit SDG 13 (Maßnahmen zum Klimaschutz) konträr entgegenstehen (Kopnina 2015). So ist festzuhalten, dass Interaktionen zwischen den einzelnen SDGs im positiven und negativen Sinn existieren, die bei der Umsetzung der Agenda 2030 zu beachten sind (Nilsson et al. 2016; Independent Group of Scienties appointed by the Secretary-General 2019).

Auch auf städtischer Ebene können unerwünschte Nebeneffekte zwischen einzelnen SDGs bzw. zwischen den Unterzielen der SDGs auftreten. Die Existenz von Zielkonflikten im Rahmen der Stadtentwicklung ist allerdings nicht SDGspezifisch, sondern eine grundsätzliche Herausforderung von Stadtpolitik und -verwaltung (vgl. Kap. 3).

Zum Kritikpunkt der mangelnden Ambitionen lässt sich konstatieren, dass die mit den SDGs anvisierten Ziele zu wenig ambitioniert sind, um die globalen Nachhaltigkeitsprobleme insbesondere im ökologischen Bereich bis $2030 \mathrm{zu}$ beheben (Hickel 2020). Angesichts der fortschreitenden Klimaerwärmung und der Notwendigkeit, das globale Wirtschaftssystem in den nächsten Jahrzehnten vollständig auf eine $\mathrm{CO}_{2}$-freie Wirtschaft umzustellen, reicht der mit der Agenda 2030 und den SDGs verfolgte Anspruch nicht aus. In Bezug auf den Klimawandel existiert mit SDG 13 zwar ein eigenes SDG, in dem Klimaschutzmaßnahmen als Ziele genannt werden; im Vergleich zum Übereinkommen von Paris zum Klimawandel (Paris Agreement on Climate Change), einem 2015 beschlossenen Vertrag der Klimarahmenkonvention der Vereinten Nationen (UNFCCC), werden in SDG 13 jedoch keine konkreten Vorgaben z. B. zur Einsparung von CO2-Emissionen gemacht. Die SDGs verweisen auf die Zuständigkeit von UNFCCC, konkrete Klimaziele und -maßnahmen auf globaler Ebene zu verhandeln und enthalten daher keine messbaren Zielwerte zur Dekarbonisierung.

Auch die generelle Wirksamkeit der Agenda 2030 wird, als dritter Kritikpunkt, zum Teil infrage gestellt. Die Freiwilligkeit bei der Umsetzungsarchitektur der SDGs und die Tatsache, dass es keine Sanktionsmechanismen für die Länder gibt, die keine Berichte an das HLPF liefern bzw. keinen Fortschritt bei der Umsetzung der SDGs verzeichnen, wirft Fragen nach Verbindlichkeit und Schlagkraft auf.

Ein anderer Einwand in Bezug auf die fehlende Wirksamkeit der SDGs ist ihre starke Orientierung an Indikatoren und Monitoringsystemen. So lässt sich zwar 
messen was für Veränderungen notwendig sind, um gesteckte Ziele zu erreichen und welche der SDG-Indikatoren bislang nicht erfüllt sind; wie diese Veränderungen und die dafür in den Indikatoren beschriebenen Zielwerte erreicht werden, liegt jedoch in der individuellen Verantwortung der Länder bzw. Kommunen (Kaika 2017). In diesem Kontext ergeben sich insbesondere Herausforderungen für die Kommunen (vgl. auch Abschn. 3.2 und 3.3).

Für die SDGs kann daher, ähnlich wie bei der Umsetzung des Übereinkommens von Paris zum Klimawandel, von zwei Diskrepanzen in Bezug auf die Ambitionen und die Implementierung gesprochen werden. Die Diskrepanzen liegen einerseits in den zu wenig ambitionierten Zielsetzungen der SDGs insbesondere bei den Einsparungen von $\mathrm{CO}_{2}$-Emissionen (Ambition Gap) und andererseits in der bislang nur schleppend stattfindenden Umsetzung der ohnehin schon wenig anspruchsvollen Ziele (Implementation Gap).

Die Kritikpunkte an den SDGs zeigen, dass die Agenda 2030 kein Allheilmittel dafür ist, die Welt zu mehr Nachhaltigkeit zu transformieren. Vor dem Hintergrund schwacher Wirksamkeit internationaler Vertragsabkommen spielt daher die Frage, wie und mit welchen Mitteln die SDGs auf Ebene der Städte interpretiert und umgesetzt werden, eine wichtige Rolle (mehr dazu in Kap. 3).

\subsection{Stand der Umsetzung}

Mehr als fünf Jahre nach der Verabschiedung der Agenda 2030 und der SDGs eröffnen verschiedene Dokumente einen Blick auf den Stand der Umsetzung und den erkennbaren Fortschritten in Bezug auf eine nachhaltige Entwicklung.

Auf globaler Ebene wird die Umsetzung der Agenda 2030 und der SDGs offiziell durch den Fortschrittsbericht (Progress Report) des Generalsekretärs der Vereinten Nationen analysiert (United Nations 2020). Im jüngsten SDG Progress Report wird konstatiert, dass in einigen Bereichen Fortschritte erzielt wurden. Genannt wird beispielsweise der zwischen 2015 und 2020 weltweit gestiegene Zugang zu Elektrizität oder der höhere Anteil von Frauen in Regierungen. Fortschritte sind auch in der Bekämpfung von Armut und dem Zugang zu Trinkwasser erkennbar. Der Bericht nimmt zudem auf die COVID-19 Pandemie Bezug und erläutert, dass diese Einfluss auf alle SDGs hat und die Erreichung der Ziele erschweren kann.

Global gesehen zeichnet der Progress Report ein eher düsteres Bild zum Umsetzungsstand der Agenda 2030 auf: Nur wenige Maßnahmen in Bezug auf den Klimawandel wurden angegangen; die Versauerung der Ozeane schreitet voran, ebenso wie der Verlust der Biodiversität. Auch für das SDG 11 sind 
viele Entwicklungen negativ. Der Anteil der in Slums lebenden Personen ist nach Jahrzehnten des Rückgangs erstmalig wieder auf $24 \%$ angestiegen; nur die Hälfte der weltweiten urbanen Bevölkerung hat angemessenen Zugang zu öffentlichem Personennahverkehr. Die Luftqualität hat sich hingegen im Jahr 2020 in vielen Städten verbessert (United Nations 2020). Dies könnte allerdings ein temporärer Effekt sein, der auf dem Rückgang der wirtschaftlichen Produktion in Zeiten von COVID-19 beruht. Zusammenfassend kann von einer Diskrepanz zwischen Rhetorik und Handeln gesprochen werden. Die UN Mitgliedstaaten heben zwar grundsätzlich die Bedeutung der SDGs hervor, konkrete Veränderungen sind jedoch bisher nur in geringem Maße zu erkennen (UCLG 2019, S. 79).

Der globale Entwicklungsbericht (Global Sustainable Development Report), der 2019 von einem durch den Generalsekretär der Vereinten Nationen berufenen Team von Wissenschaftlerinnen und Wissenschaftlern erstellt wurde, sieht die Umsetzung der SDGs ebenfalls auf keinem guten Weg (Independent Group of Scientists appointed by the Secretary-General 2019). Insbesondere die steigende globale Ungleichheit, der Klimawandel, der Verlust der Biodiversität und das zunehmende Abfallaufkommen werden kritisch gesehen. In diesen Themenfeldern ist ein Trend hin zu einer Verschlechterung der Situation festzustellen. Allerdings sieht der Global Sustainable Development Report auch optimistisch stimmende Entwicklungen. Sechs sogenannte Einstiegspunkte werden beschrieben, in denen eine Transformation zu Nachhaltigkeit besonders vielversprechend ist, da hier Veränderungen schnell und mit großer Wirkung durchgeführt werden können. Als einer dieser Einstiegspunkte wird die urbane und peri-urbane Entwicklung gesehen, da aufgrund des hohen Anteils der Stadtbevölkerung an der Weltbevölkerung urbane Maßnahmen zu mehr Nachhaltigkeit positive Effekte im globalen Maßstab haben und durch und mit den Stadtbewohner*innen ein besonderes Potenzial zur Umsetzung von Prozessen und Maßnahmen gegeben ist.

Informationen zum Stand der Umsetzung auf nationaler Ebene bieten die bereits erwähnten freiwilligen Berichte (VNRs) für das HLPF. Vergleicht man die Berichte wird deutlich, wie unterschiedlich die Umsetzung in den einzelnen Ländern angegangen wird und wie verschieden die Prioritäten in Bezug auf die SDGs sind. Deutschland hat im Jahr 2016 einen ersten VNR verfasst und wird im Jahr 2021 einen weiteren veröffentlichen. Parallel dazu wurde mit der 2016 erschienenen und 2018 überarbeiteten Deutschen Nachhaltigkeitsstrategie ein Dokument erstellt, in dem die Umsetzung der SDGs in Deutschland dargestellt wird (Bundesregierung 2018). Die Nachhaltigkeitsstrategie, für die eine erneute Weiterentwicklung im März 2021 erschienen ist (Bundesregierung 2021), adressiert drei unterschiedliche Maßnahmentypen: 
- Maßnahmen mit Wirkung in Deutschland (d. h. innerhalb der Grenzen Deutschlands)

- Maßnahmen durch Deutschland (d. h. Effekte, die durch Entwicklungen innerhalb Deutschlands außerhalb Deutschlands auftreten)

- Maßnahmen mit Deutschland (d. h. Maßnahmen, die von Deutschland z. B. im Rahmen der Entwicklungszusammenarbeit außerhalb Deutschlands gefördert werden).

\section{- Linksammlung zum Stand der Umsetzung der SDGs}

- Liste mit den bislang veröffentlichten Voluntary National Reports: https://sustainabledevelopment.un.org/vnrs/

- Deutsche Nachhaltigkeitsstrategie (Weiterentwicklung 2021) https://www.bundesregierung.de/breg-de/aktuelles/nachhaltigkeits strategie-2021-1873560

- Report des UN Generalsekretärs zum Fortschritt in der Umsetzung der SDGs: https://unstats.un.org/sdgs/report/2020/The-SustainableDevelopment-Goals-Report-2020.pdf

- Global Sustainable Development Report von Wissenschaftlerinnen und Wissenschaftlern https://sustainabledevelopment.un.org/ content/documents/24797GSDR_report_2019.pdf

Zusammenfassend lässt sich festhalten, dass sich viele mit der Agenda 2030 verknüpfte Erwartungen sowohl auf nationaler als auch auf internationaler Ebene bislang nicht oder nur in geringem Maße erfüllt haben. Dem übergeordneten Anspruch der Agenda, unsere Welt zu transformieren, werden die bisherigen Entwicklungen nicht gerecht. Die Umsetzungsarchitektur, die auf rechtlich nicht verbindliche multilaterale Verträge und freiwillige nationale Berichterstattung setzt, ist nicht ausreichend.

In Bezug auf die Umsetzung der Agenda 2030 werden große Erwartungen an die Städte formuliert. Es wird davon ausgegangen, dass Transformationen zu mehr Nachhaltigkeit in den Städten einfacher und schneller zu realisieren sind als auf nationaler oder globaler Ebene (Independent Group of Scientists appointed by the Secretary-General 2019) und Städte somit entscheidend für die Erreichung der globalen Nachhaltigkeitsziele sind (vgl. hierzu auch Angelo und Wachsmuth 2020). Das folgende Kapitel geht daher auf verschiedene urbane Herausforderungen bei der Umsetzung der SDGs und die Entwicklung der nachhaltigen Stadtentwicklung im Speziellen ein. 
Open Access Dieses Kapitel wird unter der Creative Commons Namensnennung 4.0 International Lizenz (http://creativecommons.org/licenses/by/4.0/deed.de) veröffentlicht, welche die Nutzung, Vervielfältigung, Bearbeitung, Verbreitung und Wiedergabe in jeglichem Medium und Format erlaubt, sofern Sie den/die ursprünglichen Autor(en) und die Quelle ordnungsgemäß nennen, einen Link zur Creative Commons Lizenz beifügen und angeben, ob Änderungen vorgenommen wurden.

Die in diesem Kapitel enthaltenen Bilder und sonstiges Drittmaterial unterliegen ebenfalls der genannten Creative Commons Lizenz, sofern sich aus der Abbildungslegende nichts anderes ergibt. Sofern das betreffende Material nicht unter der genannten Creative Commons Lizenz steht und die betreffende Handlung nicht nach gesetzlichen Vorschriften erlaubt ist, ist für die oben aufgeführten Weiterverwendungen des Materials die Einwilligung des jeweiligen Rechteinhabers einzuholen.

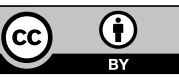

